\title{
Strategies for Optimizing Heavy Metal Staining in Freeze-substituted Resin Embedded Plants Samples for Electron and X-ray Microscopy
}

\author{
Kirk Czymmek, Keith Duncan, Ningning Zhang and Ru Zhang \\ Donald Danforth Plant Science Center, Saint Louis, Missouri, United States
}

The application of physical fixation using cryo-techniques for plant samples has distinct advantages for the rapid immobilization of dynamic sub-cellular structures and circumvents many of the challenges faced with the plant cell wall, a potent barrier to conventional chemical fixation methods. Over the last decade, numerous protocol developments have provided incremental improvements of well-established freezesubstitution fixation routines, including the enhancement of cell membranes by inclusion of water [1], compatibility with fluorescent proteins and super-resolution microscopy [2] and rapid freeze-substitution [3], to name a few. Concomitantly, advances in 3D imaging techniques, such as serial block-face scanning electron microscopy (SBF-SEM) [4], [5] and x-ray microscopy (XRM) [6], have increasingly provided critical insights into complex and multi-dimensional biological structures, including plants [7], [8].

Both SBF-SEM and XRM depend on high atomic number contrast agents for improved throughput, resolution and overall image contrast. While freeze-substituted resin-embedded plant samples are compatible with, and benefit greatly from metal enhancement for SBF-SEM [9], a paucity of data exists with cryo-fixation for SBF-SEM. Further optimization is possible and the same protocols can be effectively and interchangeably used for electron and x-ray microscopy studies. In this work, we used a Leica EM ICE high-pressure freezer to prepare various plant-based structures, (ie. leaf, anther, Chlamydomonas) followed by freeze-substitution and processing in solvent-based variations of heavy metal fixation regimes, typically used for conventional serial-block-face scanning electron microscopy [10]. This included the use of potassium ferrocyanide, osmium-thiocarbohydrazide-osmium (OTO), lead and uranium salts and exploring the impact of solvents on solubility and staining performance for resin embedded samples. Our results will compare and contrast the different staining strategies applied and demonstrate the utility of increased metalization toward high-fidelity cryogenic cell preservation coupled to high-resolution 3D correlative XRM to EM workflows.

\section{References}

[1] P. Walther and A. Ziegler, "Freeze substitution of high-pressure frozen samples: The visibility of biological membranes is improved when the substitution medium contains water," J. Microsc., vol. 208, no. 1, pp. 3-10, 2002.

[2] E. Johnson, E. Seiradake, E. Y. Jones, I. Davis, K. Grünewald, and R. Kaufmann, "Correlative inresin super-resolution and electron microscopy using standard fluorescent proteins," Sci. Rep., vol. 5, pp. $1-8,2015$.

[3] K. L. Mcdonald and R. I. Webb, "Freeze substitution in 3 hours or less," J. Microsc., vol. 243 , no. 3, pp. 227-233, 2011.

[4] T. J. Deerinck, T. M. Shone, E. A. Bushong, R. Ramachandra, S. T. Peltier, and M. H. Ellisman, "High-performance serial block-face SEM of nonconductive biological samples enabled by focal gas injection-based charge compensation," J. Microsc., vol. 270, no. 2, pp. 142-149, 2018.

[5] E. A. Bushong et al., "X-ray Microscopy as an Approach to Increasing Accuracy and Efficiency of Serial Block-face Imaging for Correlated Light and Electron Microscopy of Biological Specimens," Microsc. Microanal., vol. 21, no. 1, pp. 231-238, 2015. 
[6] M. Kittelmann, C. Hawes, and L. Hughes, "Serial block face scanning electron microscopy and the reconstruction of plant cell membrane systems," J. Microsc., vol. 263, no. 2, pp. 200-211, 2016.

[7] J. Jeiter, Y. M. Staedler, J. Schönenberger, M. Weigend, and F. Luebert, "Gynoecium and fruit development in Heliotropium sect. Heliothamnus (heliotropiaceae)," Int. J. Plant Sci., vol. 179, no. 4, pp. 275-286, 2018.

[8] K. Czymmek, A. Sawant, K. Goodman, J. Pennington, P. Pedersen, M.Hoon, and M.S. Ortegui. "Imaging Plant Cells by High-pressure Freezing and Serial Block-Face Scanning Electron Microscopy," in Springer Protocols, Plant Endosomes, M. S. Ortegui, Ed. Springer US, 2020, p. In Press.

[9] Y. Hua, P. Laserstein, and M. Helmstaedter, "Large-volume en-bloc staining for electron microscopy-based connectomics," Nat. Commun., vol. 6, pp. 1-7, 2015. 